\title{
Inhalt des 203. Bandes.
}

\author{
Erstes Heft (5. Januar).
}

Seite

I. Strada, Ferdinando, Beiträge zur Kenntnis der Geschwülste der Hypophyse und der Hypophysengegend. (Aus dem Pathologischen Institut der Universität Straßburg.) (Hierzu Taf.I und 2 Textfiguren.)

II. Kawashima, K., Uber einen Fall von multiplen Hautfibromen mit Nebennierengeschwulst. Ein Beitrag zur Kenntnis des sog. Morbus Recklinghausen. (Aus dem Pathologischen Institute der Universität Berlin.) (Hierzu Taf. II.) . . . . . . . . . . . . . . . . .

III. Goldzieher, Max, und v. Bókay, Zoltán, Der primäre Leberkrebs. (Aus dem II. Pathologisch-anatomischen Institute der Universität in Budapest.) (Hierzu 10 Textfiguren.) . . . . . . . .

IV. StraBberg, Max, Uber heterotope Knochenbildungen in der Haut. (Aus dem Pathologisch-anatomischen Universitätsinstitut in Wien.) (Hierzu 12 Textfiguren.) . . . . . . . . . . . . .

V. Zuckermann, Hans, Uber ein knochenhaltiges Lipom am Tuber cinereum. (Aus dem Pathologisch-anatomischen Institute der Universität Wien.) (Hierzu 2 Textfignren.) . . . . . . . 15̄7

\section{Zweites Heft (2. Februar).}

V. Zuckermann, Hans, Ober ein knochenhaltiges Lipom am Tuber cinereum. (Aus dem Pathologisch-anatomischen Institute der Universität Wien.) (SchluB.) . . . . . . . . . . . . . . 161

VI. Hecht, B., Ein Lipom der Pia mater cerebralis. (Aus dem Pathologischen Institute der Universität Kiel.) (Hierzu 1 Textfigur.) . . 165

VII. Ciechanowski, St., Ober Darmemphysem. (Aus dem Institute für pathologische Anatomie der k. k Jagellonischen Universität Krakan.) (Hierzu 12 Textfiguren.) . . . . . . . . . . . . . . . . 170

VIII. B olognesi, Giuseppe, Der Verschluß der MesenterialgefäBe. Kritische Betrachtungen und experimentelle Untersuchungen. (Aus der chirurgischen Klinik der Universität Modena.) (Hierzu Taf. III. IV.)

IX. Lederer, Richard, Uber eine angeborene membranöse Verengung der pars prostatica urethrae. (Aus der Prosektur des k. k. Kaiser Franz Joseph-Spitales in Wien.) (Hierzu 2 Textfiguren.) . . . . .

X. Schilder, Paul, Uber Mißbildungen der Schilddrüse. (Aus dem Pathologisch-anatomischen Institute der Universität Wien.) (Hierzu Taf. V und 9 Textfiguren.) . . . . . . . . . . . . . 246 
XI. Haberfeld, Walther. Die Epithelkörperchen bei Tetanie und bei einigen anderen Erkrankungen. (Aus dem Pathologisch-anatomischen Universitätsinstitute in Wien.) (Hierzu 8 Textfiguren.) . . . . . 289

\section{Drittes Heft (7. März).}

Xl. Haberfeld, Walther, Die Epithelkörperchen bei Tetanie und bei einigen anderen Erkrankungen. (Aus dem Pathologisch-anatomischen Universitätsinstitute in Wien.) (SchluB.) . . . . . . . . . 321

XII. Ramberg, Marie, Beobachtungen über Glykogen in der Thyreoidea. (Aus dem Patholog. Institut der Universität Bern.) (Hierzu Taf. VI.) 348

XIII. Otto, C., Uber Arteriosklerose bei Tieren und ihr Verhältnis zur menschlichen Arteriosklerose. (Aus dem Anatomisch-pathologischen Institut der Warschauer Universität.) (Hierzu Taf. VII.) . . . . . 352

XIV. William, N., und A brik os s off, A., Ein Herz mit linkem Doppelvorhof. (Aus der inneren Abteilung und der Prosektur des städtischen Marosoffschen Kinderkrankenhauses in Moskau.) (Hierzu Taf. VIII.). .

XV. Abrikossoff, A., Aneurysma des linken Herzventrikels mit abnormer Abgangsstelle der linken Koronararterie von der Pulmonalis bei einem fünfmonatlichen Kinde. (Hierzu 5 Textfiguren.) . . . . . . . . .

XVI. Kawamura, R., Beiträge zur Frage der Epithelmetaplasie. (Aus dem Patholog. Institut der Universität Berlin.) (Hierzu Tafel IX.) .

XVII. Moser, Ernst, Die ersten Veränderungen der Nierentuberkulose bei den Schlacht-Rindern. (Hierzu 6 Textfiguren.) . . . . . . . 434

XVIII. Joest, E., Untersuchungen über den Fettgehalt tuberkulöser Herde. Zugleich ein Beitrag zur Frage der nekrobiotischen Fettinfiltration. (Aus dem Pathologischen Institut der Kgl. Tierärztlichen Hochschule zu Dresden.) (Hierzu Tafel X.) . . . . . . . . . . . .

XIX. Beneke, R., und Namba, K., Ein Fall von traumatisch bedingtem Nierentumor, mit Bemerkungen zur Pathologie des ninfiltrierenden Nierenkrebses". (Aus dem Pathologisch-anatomischen Institut der Universität Marburg.) (Hierzu Tafel XI.) . . . . . . . . . . 463 